\title{
APPLICATIONS OF ONE- AND TWO-DIMENSIONAL VOLTERRA INEQUALITIES IN DIFFERENTIAL EQUATIONS OF THE HYPERBOLIC TYPE
}

\author{
LECHOSŁAW HACIA
}

Received 14 August 2002

\begin{abstract}
Some variants of one-dimensional and two-dimensional integral inequalities of the Volterra type are applied to study the behaviour properties of the solutions to various boundary value problems for partial differential equations of the hyperbolic type. Moreover, new types of integral inequalities for one and two variables, being a generalization of the Gronwall inequality, are presented and used in the theory of nonlinear hyperbolic differential equations.
\end{abstract}

2000 Mathematics Subject Classification: 26D10, 26D15.

1. Introduction. In this paper, the inequalities of the Volterra type are used to study the behaviour properties of the solutions to boundary value problems for various types of hyperbolic equations. Section 2 is concerned with fundamental remarks for one-dimensional Volterra integral equations based on the theory integral equations. The presented estimates given under weaker assumptions are applied in Section 3 to study a boundedness of solutions for linear hyperbolic equations. In Section 4, new integral inequalities of the special type are considered and applied in Section 5 to boundary value problems for nonlinear partial differential equations of the hyperbolic type.

2. Note on one-dimensional integral inequalities. Consider the following integral inequality of the Volterra type:

$$
u(x) \leq f(x)+\int_{0}^{x} k(x, s) u(s) d s .
$$

The purpose of this section is to estimate various variants to the considered inequality if the continuous functions $f$ and $u$ are assumed arbitrary (generally they are supposed to be nonnegative $[1,3,5]$ ).

Using theory of Volterra integral equations (see [2, 5]), we can get the following known results. We denote $\mathbb{R}_{+}=[0, \infty)$ and $T=\{(x, s): 0 \leq s \leq x<\infty\}$.

LEMMA 2.1. Let $f$ and $k$ be continuous functions on $\mathbb{R}_{+}$and $T$, respectively. If $k$ is a nonnegative function in $T$, then for a continuous function $u$ in $\mathbb{R}_{+}$ 
satisfying inequality (2.1), one of the estimates

$$
\begin{aligned}
& u(x) \leq f(x)+\int_{0}^{x} r(x, s) f(s) d s, \\
& u(x) \leq \bar{f}(x)\left[1+\int_{0}^{x} r(x, s) d s\right],
\end{aligned}
$$

follows, where

$$
\bar{f}(x)=\sup _{0 \leq s \leq x} f(s)
$$

and $r$ is a resolvent kernel of the form

$$
r(x, s)=\sum_{n=1}^{\infty} k_{(n)}(x, s),
$$

where $k_{(n)}$ are iterated kernels constructed by the formulas

$$
\begin{aligned}
k_{(n)}(x, s) & =\int_{s}^{x} k_{(1)}(x, \xi) k_{(n-1)}(\xi, s) d \xi \\
& =\int_{s}^{x} k_{(n-1)}(x, \xi) k_{(1)}(\xi, s) d \xi, \quad n=2,3, \ldots, \\
k_{(1)}(x, s) & =k(x, s) .
\end{aligned}
$$

If $f$ is a nondecreasing function, then

$$
u(x) \leq f(x)\left[1+\int_{0}^{x} r(x, s) d s\right] .
$$

LEMMA 2.2. If $a$ is continuous in $\mathbb{R}_{+}$, then

$$
1+\int_{0}^{x} a(s) \exp \left[\int_{s}^{x} a(\tau) d \tau\right] d s=\exp \left[\int_{0}^{x} a(s) d s\right] .
$$

Proof. Let

$$
F(s)=\exp \left[\int_{s}^{x} a(\tau) d \tau\right] \text { for arbitrary } x \in \mathbb{R}_{+},
$$

we notice that

$$
\begin{aligned}
F(x) & =1, \quad F(0)=\exp \left[\int_{0}^{x} a(\tau) d \tau\right] \\
\frac{d F}{d s} & =\exp \left[\int_{s}^{x} a(\tau) d \tau\right] \frac{d}{d s}\left[\int_{s}^{x} a(\tau) d \tau\right] \\
& =F(s)[a(s)(-1)+a(x) 0]=-a(s) F(s) .
\end{aligned}
$$


Integrating equality (2.9) over the interval $[0, x]$, we obtain

$$
\begin{gathered}
\int_{0}^{x} \frac{d F}{d s} d s=-\int_{0}^{x} a(s) F(s) d s, \\
F(x)-F(0)=-\int_{0}^{x} a(s) \exp \left[\int_{s}^{x} a(\tau) d \tau\right] d s .
\end{gathered}
$$

Hence,

$$
\begin{gathered}
1-\exp \left[\int_{0}^{x} a(\tau) d \tau\right]=-\int_{0}^{x} a(s) \exp \left[\int_{s}^{x} a(\tau) d \tau\right] d s, \\
1+\int_{0}^{x} a(s) \exp \left[\int_{s}^{x} a(\tau) d \tau\right] d s=\exp \left[\int_{0}^{x} a(\tau) d \tau\right] .
\end{gathered}
$$

THEOREM 2.3. Let $b, f$, and $u$ be continuous in $\mathbb{R}_{+}$. If $b$ is nonnegative and $u$ satisfies the inequality

$$
u(x) \leq f(x)+\int_{0}^{x} b(s) u(s) d s
$$

then

$$
u(x) \leq f(x)+\int_{0}^{x} b(s) \exp \left[\int_{s}^{x} b(\tau) d \tau\right] f(s) d s
$$

or

$$
u(x) \leq \bar{f}(x) \exp \left[\int_{0}^{x} b(s) d s\right] .
$$

Moreover, if $f$ is nondecreasing, then

$$
u(x) \leq f(x) \exp \left[\int_{0}^{x} b(s) d s\right] .
$$

Proof. It follows from Lemma 2.1 that if $k(x, s)=b(s)$, we get (see [3, 5])

$$
r(x, s)=b(s) \exp \left[\int_{s}^{x} b(\tau) d \tau\right] .
$$

Using Lemma 2.2, the proof is finished.

THEOREM 2.4. Let $a, b$ be continuous and nonnegative functions in $T$. If $a$ continuous function $u$ in $\mathbb{R}_{+}$satisfies the inequality

$$
u(x) \leq f(x)+\int_{0}^{x} a(x) b(s) u(s) d s,
$$

then for a continuous function $f$ in $\mathbb{R}_{+}$, one of the following estimates hold:

$$
\begin{aligned}
& u(x) \leq f(x)+\int_{0}^{x} a(x) b(s) \exp \left[\int_{s}^{x} a(\tau) b(\tau) d \tau\right] f(s) d s, \\
& u(x) \leq \bar{f}(x)\left[1+\int_{0}^{x} a(x) b(s) \exp \left[\int_{s}^{x} a(\tau) b(\tau) d \tau\right] d s\right] .
\end{aligned}
$$


Proof. Putting $k(x, s)=a(x) b(s) \geq 0$ in $(2.1)$, we obtain (see $[3,5])$

$$
r(x, s)=a(x) b(s) \exp \left[\int_{0}^{x} a(\tau) b(\tau) d \tau\right] .
$$

Then by virtue of Lemma 2.1, we get estimates (2.18) or (2.19).

Proposition 2.5. If additionally $f$ is nonnegative and $a$ is nonincreasing, then the Gronwall inequality

$$
u(x) \leq \bar{f}(x) \exp \left[\int_{0}^{x} a(s) b(s) d s\right]
$$

follows. If $f$ is nondecreasing, then

$$
u(x) \leq f(x) \exp \left[\int_{0}^{x} a(s) b(s) d s\right]
$$

Proof. If, moreover, $f$ is nonnegative and $a$ is nonincreasing, then

$$
\begin{aligned}
1+\int_{0}^{x} a(x) b(s) \exp \left[\int_{s}^{x} a(\tau) b(\tau) d \tau\right] d s \\
\leq 1+\int_{0}^{x} a(s) b(s) \exp \left[\int_{s}^{x} a(\tau) b(\tau) d \tau\right] d s \\
\quad=\exp \left[\int_{0}^{x} a(s) b(s) d s\right] .
\end{aligned}
$$

From here we can write estimates (2.18) and (2.19) in the forms (2.21) and (2.22), respectively.

THEOREM 2.6. Let the assumptions of Theorem 2.4 be satisfied. If a is positive, then inequality (2.17) implies

$$
u(x) \leq a(x)\left(\frac{\bar{f}}{a}\right)(x) \exp \left[\int_{0}^{x} a(s) b(s) d s\right]
$$

If additionally $f / a$ is nondecreasing, then inequality (2.22) holds.

Proof. From (2.17), we have

$$
\frac{u(x)}{a(x)} \leq \frac{f(x)}{a(x)}+\int_{0}^{x} a(s) b(s) \frac{u(s)}{a(s)} d s .
$$

By Theorem 2.3, we obtain (2.24) and the estimate

$$
\frac{u(x)}{a(x)} \leq\left(\frac{\bar{f}}{a}\right)(x) \int_{0}^{x} a(s) b(s) d s .
$$


If $f / a$ is nondecreasing, then

$$
\left(\frac{\bar{f}}{a}\right)(x)=\left(\frac{f}{a}\right)(x)
$$

and estimate (2.22) follow.

Conclusion 2.7. Theorems $2.3,2.4$, and 2.6 are obtained for negative values of $f$ and $u$ too, but are not allowed in literature (see [1, 5]). In the twodimensional case, the presented theorems are true only for nonnegative values of $f$ (see $[1,2,4]$ ).

3. Applications of one-dimensional Volterra integral equations. The estimates (2.22) and (2.24) can be used to study the boundedness of solutions for boundary value problems of special types to hyperbolic equations.

3.1. Consider the following equation:

$$
u_{x y}(x, y)=(a(x, y) u(x, y))_{y}+g(x, y),
$$

with boundary value conditions

$$
u(x, 0)=\alpha(x), \quad u(0, y)=\beta(y),
$$

which is equivalent to the integral equation

$$
u(x, y)=f_{1}(x, y)+\int_{0}^{x} a(s, y) u(s, y) d s,
$$

where

$$
f_{1}(x, y)=\alpha(x)+\beta(y)-u(0,0)-\int_{0}^{x} a(s, 0) \alpha(s) d s+\int_{0}^{x} \int_{0}^{y} g(s, t) d t d s,
$$

with continuous functions $\alpha, \beta$ and $a, g$ in $\mathbb{R}_{+}$and $\mathbb{R}_{+}^{2}$, respectively.

To estimate a solution to (3.3), we get

$$
|u(x, y)| \leq\left|f_{1}(x, y)\right|+\int_{0}^{x}|a(s, y)||u(s, y)| d s .
$$

Treating it as a one-dimensional integral inequality with respect to the variable $x$ and using Theorem 2.3 for every $y \in \mathbb{R}_{+}$, we obtain

$$
|u(x, y)| \leq f^{1}(x, y) \exp \left[\int_{0}^{x}|a(s, y)| d s\right]
$$

where

$$
f^{1}(x, y)=\sup _{0 \leq s \leq x}\left\{\left|f_{1}(s, y)\right|\right\} \quad \text { for } y \in \mathbb{R}_{+} .
$$

COROLlaRY 3.1. If $f^{1}$ is bounded in $\mathbb{R}_{+}^{2}$ and $\int_{0}^{\infty}|a(s, y)| d s<\infty$ for every $y \in \mathbb{R}_{+}$, then a solution of boundary value problem (3.1), (3.2) is bounded. 
3.2. Similarly, the equation

$$
u_{x y}(x, y)=(a(x, y) u(x, y))_{x}+g(x, y),
$$

with

$$
u(x, 0)=\alpha(x), \quad u(0, y)=\beta(y),
$$

is reduced to the following integral equation:

$$
u(x, y)=f_{2}(x, y)+\int_{0}^{y} a(x, t) u(x, t) d t
$$

where

$$
f_{2}(x, y)=\alpha(x)+\beta(y)-u(0,0)-\int_{0}^{y} a(0, t) \beta(t) d t+\int_{0}^{x} \int_{0}^{y} g(s, t) d s d t,
$$

with continuous functions $\alpha, \beta$ and $a, g$ in $\mathbb{R}_{+}$and $\mathbb{R}_{+}^{2}$, respectively. For estimation of solution of (3.10), we obtain the following inequality:

$$
|u(x, y)| \leq\left|f_{2}(x, y)\right|+\int_{0}^{y}|a(x, t)||u(x, t)| d t .
$$

Treating it as a one-dimensional Volterra inequality with respect to the variable $y$ and using Theorem 2.3 for every $x \in \mathbb{R}_{+}$, we get the estimate

$$
|u(x, y)| \leq f^{2}(x, y) \exp \left[\int_{0}^{x}|a(s, y)| d s\right],
$$

where

$$
f^{2}(x, y)=\sup _{0 \leq t \leq y}\left\{\left|f_{2}(x, t)\right|\right\} \quad \text { for } x \in \mathbb{R}_{+} .
$$

If $\left|f_{2}\right|$ is nondecreasing with respect to $y$, then we have

$$
f^{2}(x, y)=\left|f_{2}(x, y)\right| .
$$

COROLlaRY 3.2. If $f^{2}$ is bounded in $\mathbb{R}_{+}^{2}$ and $\int_{0}^{\infty}|a(x, t)| d t<\infty$ for every $x \in \mathbb{R}_{+}$, then a solution of boundary value problem (3.8), (3.9) is bounded.

4. New types of integral inequalities for one and two variables. Consider the following integral inequalities:

$$
\begin{aligned}
& u(x, y) \leq g(x, y)+a(x, y) \int_{0}^{x} p(s, y) u(s, y) d s+\int_{0}^{x} \int_{0}^{y} q(s, t) u(s, t) d t d s, \\
& u(x, y) \leq g(x, y)+a(x, y) \int_{0}^{y} p(x, t) u(x, t) d t+\int_{0}^{x} \int_{0}^{y} q(s, t) u(s, t) d t d s,
\end{aligned}
$$


for $x, y \in \mathbb{R}_{+}$with $D=\{(x, y): x, y \geq 0\}$ and the following assumptions:

$\left(\mathrm{A}_{1}\right)$ the functions $g, p$, and $q$ are nonnegative and continuous in $D$,

$\left(\mathrm{A}_{2}\right) a$ is nonincreasing, nonnegative, and continuous in $D$.

THEOREM 4.1. Let assumptions $\left(A_{1}\right)$ and $\left(A_{2}\right)$ be satisfied. If a nonnegative and continuous function $u$ satisfies inequality (4.1), then the inequality of the Gronwall type

$$
u(x, y) \leq \bar{g}(x, y) A_{p}(x, y) \exp \left(\int_{0}^{x} \int_{0}^{y} q(s, t) A_{p}(s, t) d s d t\right)
$$

holds, where

$$
\begin{aligned}
\bar{g}(x, y) & =\sup \{g(s, t): 0 \leq s \leq x, 0 \leq t \leq y\} \\
A_{P}(x, y) & =\exp \left[\int_{0}^{x} a(s, y) p(s, y) d s\right] .
\end{aligned}
$$

Proof. Denote a function

$$
r(x, y)=\int_{0}^{x} \int_{0}^{y} q(s, t) u(s, t) d s d t
$$

which, by assumptions, is nonnegative, nondecreasing, and continuous in $D$. Then inequality (4.1) can be written in the form

$$
u(x, y) \leq g(x, y)+r(x, y)+a(x, y) \int_{0}^{x} p(s, y) u(s, y) d s
$$

Treating (4.7) as a one-dimensional integral inequality for any fixed $y \in \mathbb{R}_{+}$ and using a suitable inequality, (2.21), yield

$$
u(x, y) \leq[\bar{g}(x, y)+r(x, y)] A_{P}(x, y) .
$$

Hence, we get the following two-dimensional Volterra integral inequality:

$$
\frac{u(x, y)}{A_{P}(x, y)} \leq \bar{g}(x, y)+\int_{0}^{x} \int_{0}^{y} q(s, t) A_{P}(s, t) \frac{u(s, t)}{A_{P}(s, t)} d s d t
$$

Using classical Gronwall inequality (see [1, 2, 4]), we obtain

$$
\frac{u(x, y)}{A_{P}(x, y)} \leq \bar{g}(x, y) \exp \left[\int_{0}^{x} \int_{0}^{y} q(s, t) A_{P}(s, t) \frac{u(s, t)}{A_{P}(s, t)} d s d t\right]
$$

and next we obtain inequality (4.3).

Similarly, the following theorem can be proved.

THEOREM 4.2. If the assumptions of Theorem 4.1 are fulfilled, then inequality (4.2) implies

$$
u(x, y) \leq \bar{g}(x, y) P_{A}(x, y) \exp \left(\int_{0}^{x} \int_{0}^{y} q(s, t) P_{A}(s, t) d s d t\right),
$$


where $\bar{g}$ is defined by (4.4) and

$$
P_{A}(x, y)=\exp \left[\int_{0}^{y} a(x, t) p(x, t) d t\right] .
$$

PRoof. Introducing a function $r$, similarly as in the proof of Theorem 4.1, inequality (4.2) can be replaced by

$$
u(x, y) \leq g(x, y)+r(x, y)+a(x, y) \int_{0}^{y} p(x, t) u(x, t) d t .
$$

Treating it as a one-dimensional integral inequality for any fixed $x \in \mathbb{R}_{+}$and using Proposition 2.5, we get

$$
u(x, y) \leq[\bar{g}(x, y)+r(x, y)] P_{A}(x, y)
$$

which can be reduced to the following two-dimensional inequality of the Volterra type

$$
\frac{u(x, y)}{P_{A}(x, y)} \leq \bar{g}(x, y)+\int_{0}^{x} \int_{0}^{y} q(s, t) P_{A}(s, t) \frac{u(s, t)}{P_{A}(s, t)} d s d t
$$

By Gronwall inequality (see [1, 2, 4]), we obtain inequality (4.2).

5. Applications. Particular cases of the considered inequalities will be applied to study the behaviour properties of the solutions for the boundary values problems to nonlinear differential equations of the hyperbolic type.

Consider the following differential equations of the hyperbolic type:

$$
\begin{aligned}
& u_{x y}(x, y)=f(x, y)+(p(x, y) u(x, y))_{y}+F[x, y, u(x, y)], \\
& u_{x y}(x, y)=f(x, y)+(p(x, y) u(x, y))_{x}+F[x, y, u(x, y)],
\end{aligned}
$$

with boundary conditions

$$
u(x, 0)=\alpha(x), \quad u(0, y)=\beta(y)
$$

under the assumptions

$\left(\mathrm{H}_{1}\right) f$ and $p$ are continuous in $D$;

$\left(\mathrm{H}_{2}\right) F$ is continuous in $\Theta=\{(x, y, u): x, y \geq 0,|u|<\infty\}$ and satisfying one of the following conditions:

$$
\begin{aligned}
|F(x, y, \bar{u})-F(x, y, \overline{\bar{u}})| & \leq \varphi(x, y)|\bar{u}-\overline{\bar{u}}| \text { in } \Theta \\
|F(x, y, u)| & \leq \varphi(x, y)|u| \text { in } \Theta,
\end{aligned}
$$

for nonnegative and continuous function $\varphi$ in $D$;

$\left(\mathrm{H}_{3}\right)$ the functions $\alpha$ and $\beta$ are continuous in $D$. 
Boundary value problem (5.1), (5.3) is equivalent to the integral equation

$$
u(x, y)=g(x, y)+\int_{0}^{x} p(s, y) u(s, y) d s+\int_{0}^{x} \int_{0}^{y} F[s, t, u(s, t)] d s d t
$$

where

$$
g(x, y)=\alpha(x)+\beta(y)-u(0,0)-\int_{0}^{y} p(s, o) \alpha(s) d s+\int_{0}^{x} \int_{0}^{y} f(s, t) d s d t .
$$

From (5.7), we get

$$
|u(x, y)| \leq|g(x, y)|+\int_{0}^{x}|p(s, y)||u(s, y)| d s+\int_{0}^{x} \int_{0}^{y}|F[s, t, u(s, t)]| d s d t .
$$

Using (5.5), we obtain

$$
|u(x, y)| \leq|g(x, y)|+\int_{0}^{x}|p(s, y)||u(s, y)| d s+\int_{0}^{x} \int_{0}^{y} \varphi(s, t)|u(s, t)| d s d t .
$$

Applying Theorem 4.1, we have

$$
|u(x, y)| \leq G(x, y) P(x, y) \exp \left[\int_{0}^{x} \int_{0}^{y} \varphi(s, t) P(s, t) d s d t\right],
$$

where

$$
\begin{aligned}
& P(x, y)=\exp \left(\int_{0}^{x}|p(s, y)| d s\right), \\
& G(x, y)=\sup \{|g(s, t)|: 0 \leq s \leq x, 0 \leq t \leq y\} .
\end{aligned}
$$

In this way the following theorem is proved.

THEOREM 5.1. Suppose $\left(H_{1}\right),\left(H_{2}\right)(5.5)$, and $\left(H_{3}\right)$ are true. If $G$ and $P$ are bounded in $D$ and

$$
\int_{0}^{\infty} \int_{0}^{\infty} \varphi(s, t) P(s, t) d s d t<\infty
$$

then a solution of problem (5.1), (5.3) is bounded in D and estimated by (5.10).

Similarly, we can prove the following theorem.

THEOREM 5.2. Suppose assumptions $\left(H_{1}\right),\left(H_{2}\right)(5.5)$, and $\left(H_{3}\right)$ are true. If $H$ and $Q$ are bounded in $D$ and

$$
\int_{0}^{\infty} \int_{0}^{\infty} \varphi(s, t) Q(s, t) d s d t<\infty
$$

then a solution of problems (5.2) and (5.3) are bounded in D and it satisfies the inequality

$$
|u(x, y)| \leq H(x, y) Q(x, y) \exp \left[\int_{0}^{x} \int_{0}^{y} \varphi(s, t) Q(s, t) d s d t\right],
$$


where

$$
\begin{aligned}
& Q(x, y)=\exp \left(\int_{0}^{y}|p(x, t)| d t\right), \\
& H(x, y)=\sup \{|h(s, t)|: 0 \leq s \leq x, 0 \leq t \leq y\}
\end{aligned}
$$

with

$$
h(x, y)=\alpha(x)+\beta(y)-u(0,0)-\int_{0}^{x} p(0, t) \beta(t) d t+\int_{0}^{x} \int_{0}^{y} f(s, t) d s d t .
$$

Proof. We notice that problem (5.1), (5.3) is equivalent to the integral equation

$$
u(x, y)=h(x, y)+\int_{0}^{y} p(x, t) u(x, t) d t+\int_{0}^{x} \int_{0}^{y} F[s, t, u(s, t)] d s d t .
$$

To estimate a solution of this equation, we get the following inequality:

$$
|u(x, y)| \leq|h(x, y)|+\int_{0}^{y}|p(x, t)||u(x, t)| d t+\int_{0}^{x} \int_{0}^{y}|F[s, t, u(s, t)]| d s d t
$$

which by (5.5) reduces to the integral inequality of one and two variables

$$
|u(x, y)| \leq|h(x, y)|+\int_{0}^{y}|p(x, t)||u(x, t)| d t+\int_{0}^{x} \int_{0}^{y} \varphi(s, t)|u(s, t)| d s d t .
$$

Then Theorem 5.1 yields (5.14).

COROLLARY 5.3. Existence and uniqueness of value boundary conditions (5.3) for differential equations (5.1) and (5.2) follow from assumptions $\left(H_{1}\right),\left(H_{2}\right)(5.4)$, and $\left(\mathrm{H}_{3}\right)$.

Proof. Let $u_{1}$ and $u_{2}$ be solutions of the presented problems. Then from (5.6) and (5.17), the following integral inequalities hold:

$$
\begin{aligned}
\left|u_{1}(x, y)-u_{2}(x, y)\right| \leq & \int_{0}^{x}|p(s, y)|\left|u_{1}(s, y)-u_{2}(s, y)\right| d s \\
& +\int_{0}^{x} \int_{0}^{y} \varphi(s, t)\left|u_{1}(s, t)-u_{2}(s, t)\right| d s d t, \\
\left|u_{1}(x, y)-u_{2}(x, y)\right| \leq & \int_{0}^{y}|p(x, t)|\left|u_{1}(x, t)-u_{2}(x, t)\right| d t \\
& +\int_{0}^{x} \int_{0}^{y} \varphi(s, t)\left|u_{1}(s, t)-u_{2}(s, t)\right| d s d t .
\end{aligned}
$$

Using Theorems 4.1 and 4.2, respectively, we get uniqueness

$$
u_{1}(x, y)=u_{2}(x, y)
$$


COROLLARY 5.4 (stability of solutions). Suppose that $\left(\mathrm{H}_{1}\right),\left(\mathrm{H}_{2}\right)(5.4)$, and $\left(\mathrm{H}_{3}\right)$ are true. Then solutions of value boundary problems (5.1), (5.3) and (5.2), (5.3) are stable.

Proof. Let $g_{1}$ and $g_{2}$ be continuous functions of the form (5.7) which are depended on the boundary conditions (5.3) for solutions $u_{1}$ and $u_{2}$ to (5.6), respectively, such that $\left|g_{1}(x, y)-g_{2}(x, y)\right|<\varepsilon$ in $D$ for every $\varepsilon>0$. Then, we get

$$
\begin{aligned}
\left|u_{1}(x, y)-u_{2}(x, y)\right| \leq & \varepsilon+\int_{0}^{x}|p(s, y)|\left|u_{1}(s, y)-u_{2}(s, y)\right| d s \\
& +\int_{0}^{x} \int_{0}^{y} \varphi(s, t)\left|u_{1}(s, t)-u_{2}(s, t)\right| d s d t \\
\left|u_{1}(x, y)-u_{2}(x, y)\right| \leq & \varepsilon \exp \left[\int_{0}^{x} \int_{0}^{y} \varphi(s, t) P(s, t) d s d t\right] .
\end{aligned}
$$

Similarly, for the functions $h_{1}$ and $h_{2}$ corresponding to different boundary conditions of problems (5.2) and (5.3), the following estimate holds:

$$
\left|u_{1}(x, y)-u_{2}(x, y)\right| \leq \varepsilon \exp \left[\int_{0}^{x} \int_{0}^{y} \varphi(s, t) P(s, t) d s d t\right] .
$$

\section{REFERENCES}

[1] D. Bănov and P. Simeonov, Integral Inequalities and Applications, Mathematics and Its Applications (East European Series), vol. 57, Kluwer Academic, Dordrecht, 1992.

[2] A. Corduneanu, A note on the Gronwall inequality in two independent variables, J. Integral Equations 4 (1982), no. 3, 271-276.

[3] L. Hacia and L. Kaczmarek, On the bounds of solutions of a system of Volterra integral equations, Demonstratio Math. 28 (1995), no. 4, 945-952.

[4] _ On the boundedness of solutions for system of two-dimensional Volterra integral equations, Fasc. Math. (1998), no. 28, 43-55.

[5] R. Rabczuk, Foundations of Differential Inequalities, Państwowe Wydawnictwo Naukowe, Warsaw, 1976 (Polish).

Lechosław Hącia: Institute of Mathematics, Poznań University of Technology, Piotrowo 3A, 60-965 Poznań, Poland

E-mail address: 1hacia@math.put.poznan.p1 


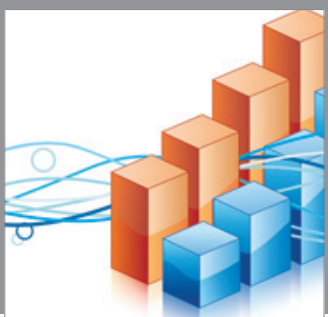

Advances in

Operations Research

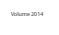

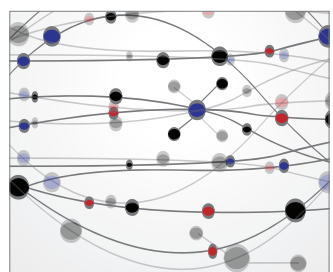

\section{The Scientific} World Journal
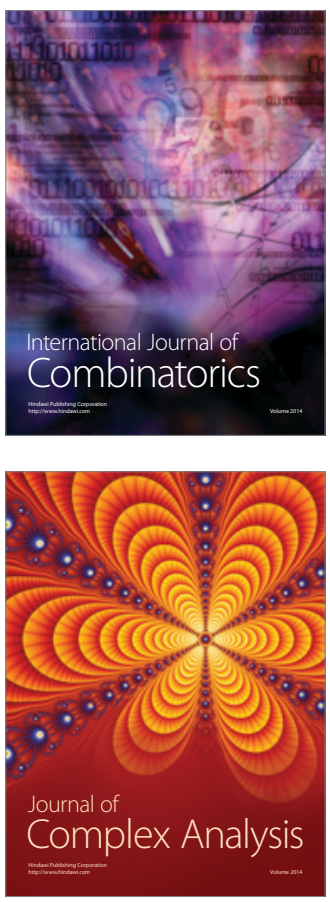

International Journal of

Mathematics and

Mathematical

Sciences
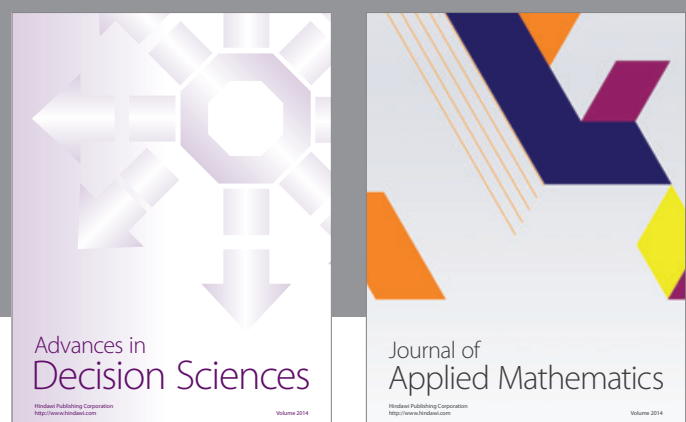

Journal of

Applied Mathematics
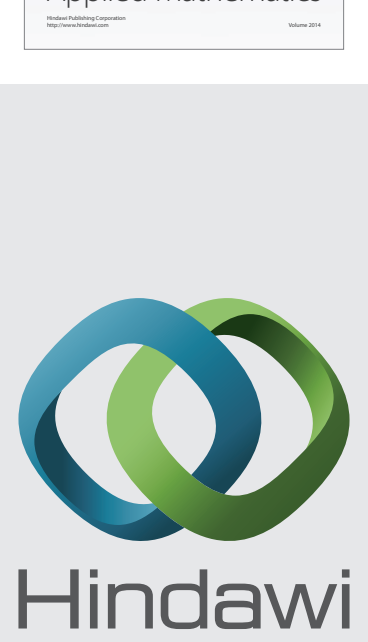

Submit your manuscripts at http://www.hindawi.com
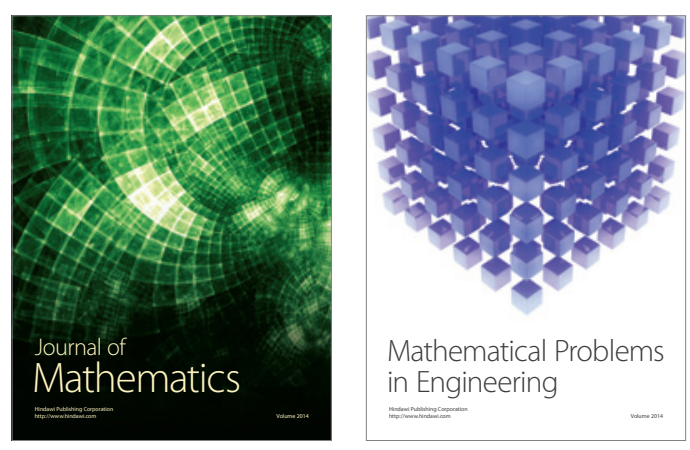

Mathematical Problems in Engineering
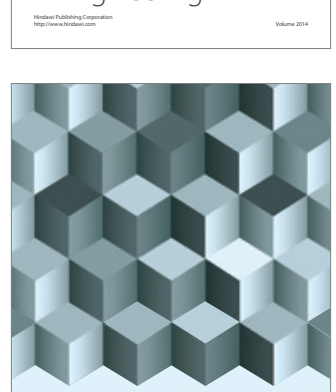

Journal of

Function Spaces
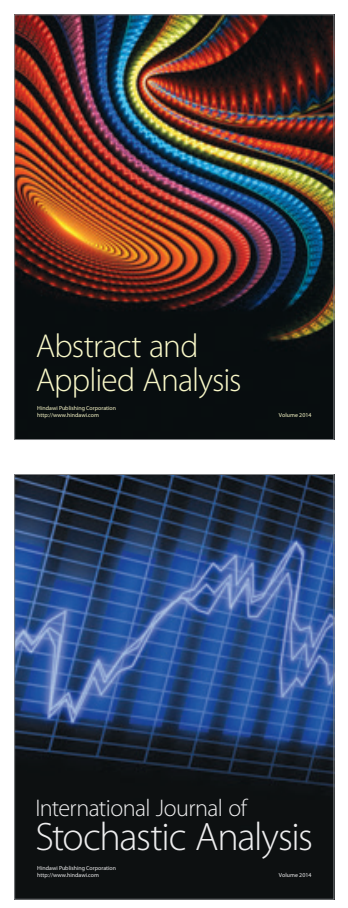

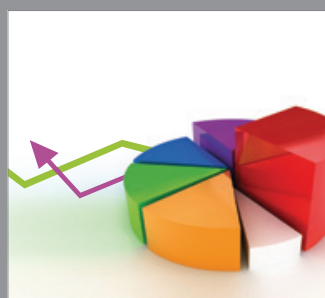

ournal of

Probability and Statistics

Promensencen
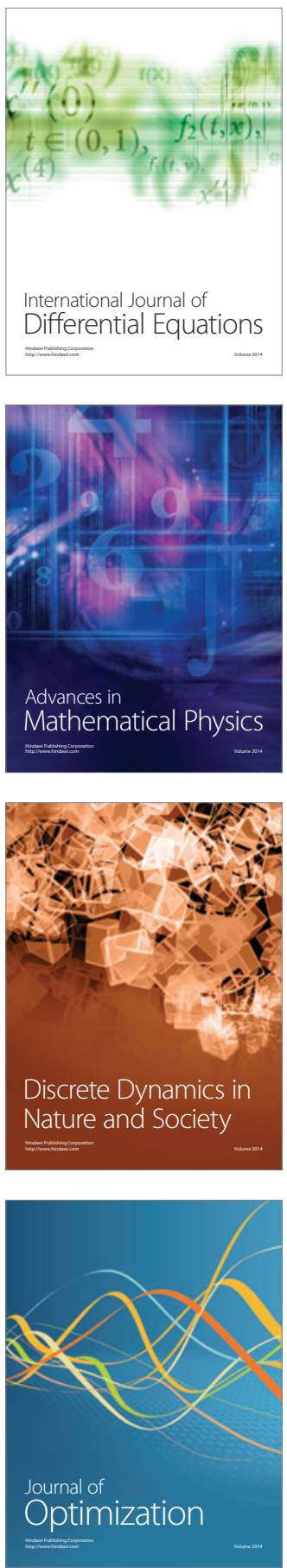